\title{
La concepción corpuscular de la materia y el concepto Mol: algunas implicaciones epistemológicas y didácticas
}

\section{Introducción}

JCSUÉ NICOLÁS MEDINA ARÉVALO', LUIS JAVIER NARVAEZ ZAMORA"

En la actualidad, uno de los aspectes fundamentales, objeto de investigación en Didáctica de la Química, es el relacionado con el aprendizaje significativo de conceptos y con la construcción de concepciones globales en el ámbito científico.

La química como ciencia está estructurada en torno a conceptos, leyes, principios y teorías, una de las cuales es la teoría atómica, directamente relacionada con la concepción corpuscular de la materia (Atomismol. como base fundamental de la estructura conceptual de la química moderna; más aún. su aceptación a principios del siglo XIX es uno de los hitos que constituye la ruptura con las concepciones precientificas. Sin embarge, el atomismo no es propiamente una idea que podemos considerar como originada en la época moderna, sino que empezó a constituir parte del acervo eultural de la humanidad desde que los filósofos jónicos lo plantearon en el sigla VI a.c. En consecuencia, es válido plantearnos los siguientes interrogantes: ¿qué condiciones específicas hicieron que el atomismo se estableciera como paradigma esencial de la química moderna?. ¿qué condiciones sociales e históricas favorecieron su aceptación?, Lqué crisis hicieron declinar a las antiguas concepciones químicas que no lo incluían en sus postulados fundamentales?

A nivel didáctico, a pesar de las múltiples investigaciones realizadas sobre la construcción de una concepción corpusculaß de la materia y sobre el aprendizaje significativo del concepto mol, éstos centinúan siendo problemas didácticos aún sin resolver. 
En este artículo se pretende argumentar que la incorporación del análisis epistemológico de la historia de las ciencias, en los procesos didácticos constituye una de las alternativas en la búsqueda de soluciones a los problemas mencionados anteriormente. El análisis epistemológico de la historia de las ciencias, permite determinar el origen de los conocimientos precientíficos, asociados con los conocimientos previos de los estudiantes, contrastarlos con los conocimientos actuales y orientar al estudiante en su apropiación de los conceptos modernos; estimular en él la actitud crítica, la creatividad y la comprensión de la función social de la actividad científica. Este análisis histórico enseña además que las ciencias no están terminadas, que están en permanente proceso de construcción y que en esa evolución, teorías que sirven para explicar los fenómenos conocidos en una época determinada, pueden ser insuficientes $y$ deficientes ante nuevos hechos y deben ser complementadas con otras de mayor poder explicativo, predictivo y de generalización.

El nivel puramente informativo o didáctico clásico podrá ser superado al incorporar la perspectiva histórica epistemológica. Desde ella, el estudiante podrá concebir a la ciencia no como algo ya hecho, dado, revelado, sino como algo que está en proceso de construcción. Podrá ver las interpretaciones como algo cambiante que depende de condiciones internas y externas; que el conocimiento no es un objeto que se compra, se vende o se transmite, sino que se construye. Podrá ver que su papel no tiene por qué ser el de un consumidor pasivo de algo ya elaborado por los "Genios", sino que debe ser el de un sujeto capaz de vincularse al proceso de construcción de la ciencia, en el cual han participado y participarán seres humanos como él.

\section{Desarrollo histórico-epistemo- lógico de la Teoría Atómica}

Desde el punto de vista histórico epistemológico, en la estructuración conceptual de la química se establece un modelo de materia en el que se caracterizan las sustancias en un nivel atómico - molecular a partir de las contribuciones realizadas desde el marco teórico o esquema conceptual de los filósofos Jónicos (siglo VI a.c.), el cual fue retomado, discutido y complementado de forma trascendental por Demócrito y Leucipo (siglo $V$ a.c.), quienes introdujeron la idea de vacío para explicar el movimiento de los átomos (Llorens, 1991), así como por Epicuro (Cubillos, et al 1989). A su vez, estos planteamientos fueron retomados y complementados por Dalton en su teoría atómica, cuyo trabajo al ser reestructurado y complementado por otros investigadores químicos se convirtió en el cimiento conceptual de la química como ciencia moderna.

Al intentar remontarnos a lo que se cree fueron las primeras ideas del concepto materia, se puede decir que el punto de partida en su construcción está situado en la escuela Jónica (siglo VI a.c.), con las doctrinas de Tales de Mileto, Anaxímenes y Anaximandro. En ella se vislumbra el paso del pensamiento mítico al pensamiento causal, al admitirse una causa natural y unidad básica de las cosas, fundamentada en la existencia de un principio común e invariable subyacente a toda transformación. Es así, como para Tales de Mileto este principio es el agua, 


\section{2}

Universidad Surcolombiana

para Anaxímenes el aire (principios de tipo común y perceptiblel y para Anaximandro el "apeiron" o lo indeterminado, una materia primera y cualitativamente indiferenciada labstracción de lo sensible a lo conceptual). El rasgo común en las ideas de estos tres pensadores es que la materia se halla de por sí en movimiento (materia vivientel, por lo que esta escuela de pensamiento se denominó hilozoismo.

\section{Origen del atomismo}

De la contradicción entre la concepción de realidad cambiante planteada por Heráclito de Efeso (550-475 a.c.). quien introduce el fuego como origen de la realidad: Ia llama simboliza el cambio incesante que se produce en todo y la concepción de realidad permanente planteada por Parménides de Elea (540 a.c.). para quien existe una materia única inmutable cualitativa y cuantitativamente indiferenciada, donde cualquier cambio en ella es aparente, surgen el "enfoque pluralista", basado en la aceptación de unos elementos y principios constituidos por materia cualitativamente diferente y el "mecanicista", en el que se interpreta todo cambio a partir de la forma, tamaño y movimiento de partículas constituidas por materia indiferenciada.

Dentro del enfoque pluralista, se destaca el pensamiento de Empédocles de Agrigento (480-430 a.c.), quien al plantear la noción de los cuatro elementos como soporte de las cualidades caliente, frío, seco y húmedo, concibe la realidad material constituida por partículas de agua, aire, fuego o tierra, siendo la proporción en que entran estos cuatro elementos, la que determinará las propiedades de las sustancias, y el de Anaxágoras, quien partiendo de la idea de Parménides de la inmutabilidad, explica el cambio admitiendo que todo preexiste en todo; sin embargo, hay que aclarar que esta concepción es incompatible con el atomismo, pues admitir que existe de todo en todo está en contradicción con la idea de la existencia de partículas formadas por una misma clase de materia.

Otra escuela de pensamiento que debemos mencionar es la Pitagórica (Pitágoras, 582-500 a.c.). uno de cuyos aportes es la introducción de un modelo conceptual basado en el número y relacionado no sólo con la idea de cantidad, sino también de forma. Se plantea aquí una concepción discontinua, discreta de la magnitud, que trasladada al mundo físico generará a través de la obra de Leucipo y Demócrito (450 a.c.) el atomismo:la materia se considera que está formada por átomos y vacío; estos átomos son sólidos, indestructibles, inmutables, infinitos en número, imperceptibles por los sentidos y no poseen cualidades. No existe una diferencia entitativa entre los átomos, es decir, no se pueden distinguir tipos de átomos según la clase de materia que los 
constituye. Las propiedades de los diferentes tipos de materia vienen dadas por la forma, tamaño y movimiento de los átomos. El aporte más notable de esta doctrina es el inicio de la orientación mecanicista en la comprensión de la materia: la explicación de las cualidades en un nivel macroscópico a partir de cambios cuantitativos que tienen lugar en un nivel microscópico.

Las ideas atomistas fueron desarrolladas luego por Epicuro (342-270 a.c.) y plasmadas en el poema de Lucrecio 19555 a.c.) De Rerum Natura, a través del cual llegaron a los siglos posteriores.

\section{EI sustancialismo, una teoría impuesta a la fuerza}

Para algunos filósofos y especialmente para Aristóteles (384-322 a.c.). la idea de una partícula de materia no divisible en otras menores, resultaba paradójica y no la aceptaron (Asimov. 1996). Aristóteles aceptó las ideas de Empédocles de los cuatro elementos. como soporte de las cuatro cualidades antes mencionadas, pero sobre este tema avanzó todavía más al afirmar que cada elemento tiene una serie de propiedades específicas que le son innatas. Así por ejemplo, es propio de la tierra el caer, mientras que en la naturaleza del fuego está el elevarse. Sin embargo, según Aristóteles, los cuerpos celestes presentan características que son diferentes de las sustancias de la tierra y que por lo tanto deberán estar formados por un quinto elemento, que llamó éter (resplandecer). Como los cielos no parecían cambiar nunca, Aristóteles considero al éter como perfecto, eterno e incorruptible. lo que lo hacía muy distinto de los cuatro elementos imperfectos de la tierra.
La teoría sustancialista (continuidad de la material de Aristóteles desarrollada e integrada a la cosmología escolástica se convirtió en el marco conceptual de referencia más importante que "dominaba" el saber durante la Edad Media y el Renacimiento, mientras que el atomismo (concepción corpuscular de la materia) fue rechazado por su relación directa con las doctrinas materialistas que se oponían a los dioses oficiales del estado. Es así, como puede explicarse que la teoría atómica fuera relegada y debiera permanecer desprestigiada durante muchos siglos a pesar de que persistía enunciada en algunos documentos que fueron accesibles a los estudiosos del tema. Sin embargo, al examinar la historia, nos demuestra que a lo largo de los siglos que separan a Demócrito de Dalton, la teoría atómica fue enseñada, elaborada, empleada, discutida y controvertida, pero jamás olvidada. Más aún, que en los años precedentes a Dalton, esta fue una de las teorías dominantes en las investigaciones cientificas.

\section{Crisis del sustancialismo y resurgimiento del atomismo}

Durante los siglos XVI y XVII d.c, la revolución copernicana conduce a una transformación total de la antigua cosmología, lo cual conlleva a una crisis a nivel de la cosmología premecanicista y sustancialista predominante hasta aquel entonces, en donde las explicaciones de tipo teológico no satisfacen la interpretación de los fenómenos naturales. Es cuando Galileo Galilei, plantea nuevas explicaciones de tipo mecanicista y matemático que satisfacen algunas inquietudes generadas en ese momento, en las cuales formuló una explicación mecánica del calor, al 
hablar del movimiento de pequeñas partículas como causa del mismo. La nueva filosofía de Descartes se ubica dentro de esta tendencia bajo la cual presenta el modelo de un mundo mecánico, detrás de cuyas apariencias subyace el movimiento de partículas. Sin embargo, para Descartes, las partículas son divisibles hasta el infinito y se hallan ubicadas en un espacio lleno totalmente por materia muy sutil.

Es Pierre Gassendi (francés), quien en el siglo XVII en abierta oposición a Descartes propone la ontología del antiguo atomismo Griego, como la que mejor se adapta al mecanicismo renacentista, en la que opone los conceptos de átomos y vacío a los de sustancia y accidente. Para Gassendi, los átomos son las sustancias reales y el vacío es simplemente el lugar o espacio en que se encuentran, permitiéndoles el movimiento. Las propiedades de los átomos según Gassendi se reducen a peso, número y medida, adaptadas a la producción de cualidades sensoriales como luz, calor, sonido y frío, entre otras. Según Cubillos y otros (1989), el gran mérito de Gassendi es haber mostrado que el atomismo griego se adaptaba mejor al tipo de explicaciones requeridas en una época sacudida por la revolución Copernicana, que la fiIosofía Aristotélica-Escolástica que había monopolizado toda la cultura Europea durante los siglos precedentes.

Es así como en los siglos XVI y XVII las ideas del atomismo comenzaron a incorporarse a la química: algunos fenómenos como la difusión del alcohol a través del papel, la condensación del vapor y el desplazamiento del cobre por el hierro en el sulfato cúprico, fueron interpretados de modo corpuscular. La principal característica de los comienzos del atomismo mecanicista es el intento de explicación de las cualidades de las sustancias a partir de la forma de los átomos. Por ejemplo, se supone que los átomos de calor son pequeños y puntiagudos, y que las uniones entre los átomos tienen lugar mediante pequeños ganchitos que estos poseen. En estas ideas puede apreciarse la asignación a los propios átomos de formas relacionadas, con base a intuiciones puramente sensoriales con las cualidades macroscópicas que pretenden explicarse. Bachelard (1975). citado por Cubillos (1989), en su obra Las intuiciones atomisticas se refiere a estas concepciones al hablar de "atomismo realista".

Conviene aclarar que la traslación de conceptos macroscópicos al mundo corpuscular se da en un momento en que no existe un concepto claro de cambio químico y no se ha desarrollado la noción de elemento químico. No existe, por tanto, la posibilidad de dar un contenido químico a la doctrina atómica; es explicable entonces que ésta fuera estéril hasta que el establecimiento por Lavoisier del concepto de elemento como sustancia simple, permitiera a Dalton conceder al átomo un significado químico preciso: ser la partícula representativa de un elemento. La relación átomo-elemento así establecida, sería un camino enormemente fructifero.

La tendencia conceptual anteriormente descrita, es una de las más comunes que se detectan en los estudiantes durante su iniciación a la Química. Esta reflexión permite formular una cuestión fundamental: ¿Cómo se produce en los estudiantes la transición desde la concepción corpuscular mecanicista que pueden construir a partir del estudio experimental del comportamiento de los gases, a una interpretación corpuscular del cambio químico y sus leyes?

En la interpretación del comportamiento mecánico de los gases, un 


\section{5}

Paideia Surcolombiana

terreno estrictamente físico, fue donde el atomismo mecanicista dio sus frutos. Así, Daniel Bernouilli propuso un modelo corpuscular de los gases que introduce aspectos básicos de lo que más tarde sería la teoría cinético molecular. Bernouilli, admitió la existencia de partículas muy pequeñas en constante agitación, que chocan entre ellas y con las paredes del recipiente de modo totalmente elástico. En este tipo de choques la energía cinética se conserva y por tanto, el movimiento es indefinido y la presión, como magnitud macroscópica, es el resultado de múltiples impactos de las partículas; a partir de esta idea logró explicar la relación $P V=$ cte, que Boyle había obtenido empíricamente. Introdujo así la notable anticipación consistente en admitir que una ley determinista, basada en la medición de magnitudes macroscópicas podía ser explicada en términos de un fenómeno aleatorio a escala corpuscular (Furió, C, 1995). Al

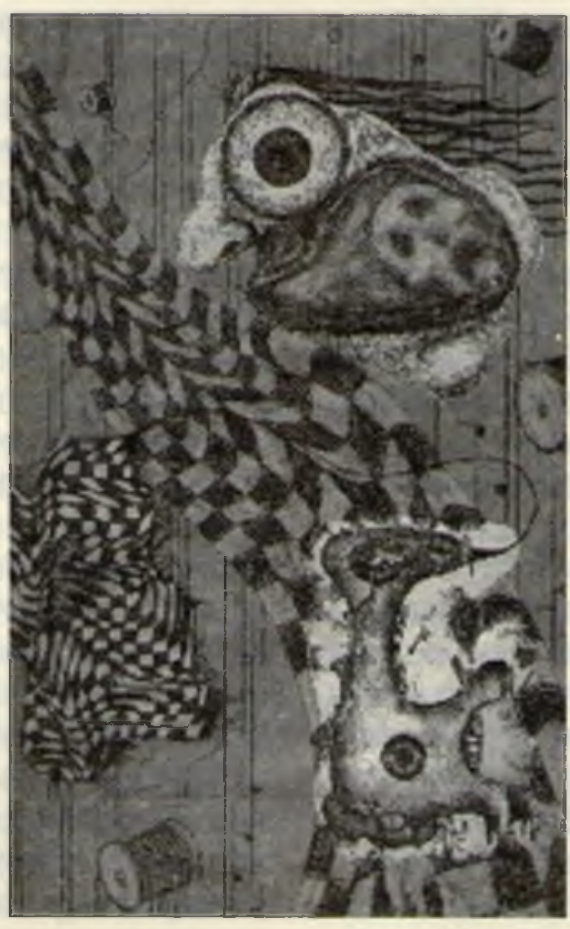

formado por corpúsculos microscópi$\cos y$ que todo fenómeno natural podía ser explicado en términos de la forma corpuscular, tamaño, movimiento e interacción de los mencionados corpúsculos. A lo largo de los siglos XVII y XVIII los científicos usaron la hipótesis atómica no sólo para explicar algunos fenómenos ópticos, químicos y neumáticos, sino también otros de la mecánica y de la teoría del calor.

En los primeros albores del siglo XIX John Dalton (1766-1844), realiza un proceso de síntesis entre la tradición pluralista y las doctrinas corpusculares. Su aporte específico consistió no en haber formulado la existencia de los átomos, hecho que como se mencionó anteriormente hacía ya parte de la filosofía de la época, sino en haber dado una formulación cuantitativa del atomismo, que sirvió de base teórica para la química de los elementos. Para Dalton, los átomos no son solamente el soporte de las cualidades diferentes que puede tener la materia, sino la base material que explica las mismo tiempo identificó el calentamiento como un movimiento más intenso de las partículas, aproximándose así al concepto termodinámico actual de la temperatura.

Según Wurts (1997), después de 1630 y particularmente luego de la aparición de las obras de Descartes y de Gassendi, la mayoría de los científicos asumieron que el universo estaba leyes cuantitativas establecidas como la de los pesos equivalentes y las proporciones definidas. Con la teoría atómica de Dalton ya no hay más principios químicos que expliquen las propiedades de las sustancias, sino elementos a los que corresponden átomos de un mismo tipo, de igual naturaleza y lo que es más interesante igual peso, habiendo tantas clases de 
átomos como elementos químicos. Dalton, relaciona el concepto operativo de elemento como sustancia simple de Lavoisier, con un tipo de átomos con unas cualidades específicas, cualidades además que dejan de ser subjetivas y relacionadas con lo sensorial, para referirse a magnitudes bien determinadas, como el peso. En efecto, a partir de éste momento, la determinación de los pesos atómicos es un objetivo en torno al cual surgen algunos aportes valiosos en la química como ciencia moderna.

La teoría atómica de Dalton tiene un poder explicativo tan amplio, que en torno a ella se estructuran las respuestas a algunos interrogantes sobre la composición de los cuerpos materiales, sobre la naturaleza de las reacciones químicas y sobre el por qué los elementos se combinan en proporciones definidas para generar compuestos, entre otras. Esta teoría tenía un gran poder predictivo al anticipar la ley de las proporciones múltiples. antes de ser encontrada experimentalmente. Lavoisier y Dalton, son las dos grandes figuras que se sitúan en el eje de esta ruptura epistemológica. La adopción de los métodos introducidos por Lavoisier y el desarrollo de sus concepciones mecanicistas, le permiten a Dalton, llevar hasta las últimas consecuencias la ruptura epistemológica iniciada por Lavoisier, el mártir de la revolución francesa.

Uno de los méritos de Dalton fue precisamente el de que, en vez de dedicarse a elaborar nuevos modelos más sofisticados dentro de la química sustancialista se decide a romper radicalmente con ella, para introducir el nuevo modo de pensar en la química: la concepción corpuscular de la materia.

Desde el punto de vista de las concepciones actuales algunas de las hi- pótesis de Dalton no son válidas. Así por ejemplo, los átomos de un mismo elemento no son necesariamente iguales (isótopos), si es posible la trasmutación de los elementos y los átomos son divisibles en partículas menores. Ahora bien, en el plano de los procesos químicos ordinarios, estas ideas son válidas.

\section{El Concepto Mol}

Basándose en la teoría atómica de Dalton. Amadeo Avogadro (1776-1856) formuló la hípotesis según la cual: "volúmenes iguales de gases diferentes, en las mismas condiciones de presión y temperatura poseen el mismo número de moléculas" (aunque no abordó el cálculo de dicho númerol. Al mismo tiempo admitió la posibilidad de que las partículas de los gases estuvieran constituidas por dos o más átomos. introduciendo el concepto de molécula.

A mediados del siglo XIX se produce una sucesión de intentos de determinación de la escala de pesos átomicos, como desarrollo de las ideas de Dalton, tarea en la que pueden destacarse las contribuciones de Berzelius. Dulong - Petit y Prout.

El resultado de todos estos intentos dio lugar a un panorama confuso, pues la tabla de Berzelius, aunque muy cercana a la actual, no era ampliamente aceptada y seguían utilizándose tablas de pesos equivalentes, cuyos valores dependían del compuesto analizado, así como de múltiples sistemas de formulación. La teoría atómica se encontraba en cierto modo, en un callejón sin salida, pues mientras la teoría de Dalton no permitía disponer de criterios totalmente válidos para calcular los pesos atómicos, tampoco se aceptaba la hipótesis de Avogadro. Ante 
esta situación Kekulé, convocó la primera reunión científica internacional de la historia. Se llamó Primer Congreso Internacional de Química y se reunió en 1860 en Karlsrune, Alemania, que había de marcar un hito en la historia de la Química. Asistieron 140 delegados, entre los que se encontraba el químico italiano Stanislao Cannizzaro (1826-1910). Dos años antes, éste. había conocido el trabajo de su compatriota Avogadro y comprendió que su hipótesis podía utilizarse para distinguir entre peso atómico y peso molecular de los elementos gaseosos importantes, y que esta distinción serviría para aclarar la cuestión de los pesos atómicos de los elementos en general. Además, comprendió la importancia de distinguir cuidadosamente el peso atómico del peso equivalente. En el congreso, Cannizzaro hizo una gran exposición del tema; sus opiniones ganaron adeptos en el mundo de la química; a partir de entonces se clarificó la cuestión del peso atómico y se apreció la importancia de la tabla de pesos atómicos de Berzelius.

Conocidos los pesos atómicos, se estableció un vínculo cuantitativo entre el mundo macroscópico y el corpuscular, y así surgió la idea de manejar cantidades de materia que tuviesen el mismo número de partículas, estableciéndose los conceptos de átomo gramo y de molécula - gramo, precursores del actual concepto de Mol. Al plantearse los conceptos de átomo gramo y de molécula - gramo, surge la conveniencia de determinar el número de átomos o de moléculas contenidos en esas porciones de materia.

Loschmit, sería quien primero lo intentaría en el año de 1865. obteniendo un valor de $4.2 \times 10^{22}$. Poco después aparecería el concepto $\mathrm{Mol}$ introduci- do por Ostwald (1896), al tiempo que surgían ásperas discusiones en torno a la aplicación, interpretación y significado de este concepto asociado a: unidad individual de masas /sinónimo de átomo - gramo, molécula - gramo, fórmula gramol o a porción de sustancia. En 1913. Mosseley, al establecer experimentalmente el concepto de número atómico, introdujo un criterio unívoco para identificar el elemento como una clase de átomos con la misma cantidad de cargas eléctricas positivas en el núcleo.

En 1958, la International Union of Pure and Applied Physics (I.U.P.A.P.), intentaría unificar criterios proponiendo el mol como: "La cantidad de sustancia que contiene el número de átomos, moléculas o iones, que el de átomos existente exactamente en $16 \mathrm{~g}$ de ${ }^{16} \mathrm{O}$ (I.U.P.A.P, 1958). No obstante la complejidad y alto grado de abstracción que presenta el concepto cantidad de sustancia (Diekers, 1996) impidió que se terminara la controversia.

En 1963, la International Union of Pure and Applied Chemistry (I.U.PA.C.). intentó superar esta dificultad al proponer el concepto Mol, como: "La cantidad de sustancia de un sistema material que contiene tantas entidades elementales como átomos hay en 1,2 X $10^{-3} \mathrm{Kg}$ de ${ }^{12} \mathrm{C}$. Al usar el concepto mol, las entidades elementales deberán especificarse, pudiendo tratarse de átomos, moléculas, iones, electrones, protones y otras partículas" (Diekers, 1996). La cantidad de sustancia es proporcional al número de entidades, siendo la constante de proporcionalidad el número de Avogadro; un mol contiene 6, $02252 \times 10^{23}$ entidades. Un mol de un elemento con masa atómica relativa $A$, tiene una masa de $A$ gramos la esta masa se le llamaba antiguamen- 
te un átomo - gramo del elementol. Un mol de un compuesto de masa molecular relativa $M$, tiene una masa de $M$ gramos la esto se llamaba una molécula gramo del compuestol.

A pesar de las complementaciones realizadas, tales como el introducir el concepto sistema material o la necesidad de especificar las entidades elementales, continuaron las discusiones en torno a la pretensión de cuantificar la cantidad de sustancia en ecuaciones matemáticas (Kolb, 1994). así como las imprecisiones señaladas de tomar el mol como una cantidad individual, por ser unidad de valor numérico distinto para diferentes sustancias /Cuestión rechazada por la I.U.P.A.C. en 1972). Así también, tomar el mol como unidad de cantidad de sustancia, muestra la necesidad de definir la magnitud cantidad de sustancia y luego transferirla a cálculos cuantitativos, lo que intentó superarse con la introducción del término número de moles (Kolb, 1994), lo cual fue criticado por la I.U.P.A.C. que consideró inconsistente llamar " $n$ " al número de moles, como lo es llamar " $\mathrm{m}$ " al número de Kilogramos.

El concepto Mol definido de forma clara por la I.U.P.A.C. en 1963, creó una fuerte controversia, quizás motivada por el carácter abstracto que el concepto cantidad de sustancia tenía, y su asociación con aspectos como masa, volumen y número. Toda esta controversia se ha dado a nivel histórico debido al enfrentamiento entre las dos concepciones existentes sobre la estructura de la materia: la concepción discontinua, empleada por la I.U.P.A.C. en la definición del mol y la concepción continua o sustancialista lla no abstracción de la concepción corpuscular de la material.
Si se analiza desde el punto de vista epistemológico y didáctico, cómo los estudiantes abordan el problema corpuscular de la materia, para Llorenz (1997) este problema es abordado por los estudiantes en razón de la existencia en ellos de un atomismo intuitivo. que da lugar al atomismo substancial (conservación sucesiva del pesol y el atomismo espacial (conservación sucesiva del volumen), los cuales son expresiones de las operaciones de adición positiva con reversibilidad y asociatividad.

No obstante, para algunos autores como Diekers (1996). García (1990), Lee (1961), Furió (1995), Novick (1996), y Llorens (1997), entre otros, el atomismo intuitivo no parece ser condición suficiente para un aprendizaje de la teoría atómico-molecular. Novick y Menis determinaron numerosas dificultades en la interpretación atómico - molecular en diferentes experiencias con estudiantes, incluso en los mejor dotados. García, Diekers y Furió, determinaron en estudiantes con edades comprendidas entre 15 y 18 años, la asignación de características macroscópicas a entidades atómico-moleculares. En este sentido, puede admitirse la existencia de una acomodación de la interpretación corpuscular a un esquema conceptual basado en la interpretación macroscópica y directamente perceptible.

Llorens al estudiar el desarrollo de los conceptos de estructura y el proceso de aprendizaje de la teoría atómico - molecular a partir de cuatro niveles de descripción (fenomenológico, atómico, molecular y multiatómico), determinó que las dificultades en el aprendizaje de los conceptos químicos, residen en la naturaleza abstracta de los conceptos involucrados y en la exigencia de operativizar variables entre los fenómenos. 


\section{9}

Paideia Surcolombiana

\section{Conclusiones implícitas}

Si entendemos al conocimiento como un proceso a través del cual el ser humano aprehende el mundo, su constitución y funcionamiento; entonces, los procesos educativos en ciencias, requieren de la dimensión temporal y dinámica que les proporciona el análisis epistemológico de su desarrollo histórico.

- Si se tiene en cuenta que un concepto está estructurado por un conjunto de nociones que lo cualifican como tal (atributos de criterio), y que si ese concepto tiene un alto grado de exigencia cognitiva que lo caracteriza como una categoría (como es el caso del moll, sus nociones, por tanto, se discriminarán como conceptos (conceptos estructurantes) cualificados, cada uno de ellos por un conjunto de nociones. Esto implica que para lograr el aprendizaje significativo del concepto (categoría) Mol, se requiere en primera instancia el aprendizaje significativo de los conceptos estructurantes de esta categoría.

- Los conceptos de sustancia, elemento, compuesto, cantidad de sustancia, mol y cambio químico, quedan perfectamente establecidos y delimitados cuando se incorporan desde el punto de vista de la concepción corpuscular de la materia. La claridad de la teoría atómica debe ser explota-

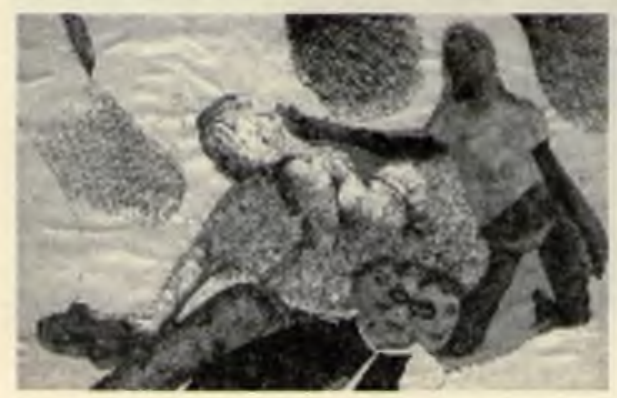

da didácticamente reinterpretando a partir de ella el conocimiento experimental previo, aproximando así a los estudiantes al significado de una teoría científica.

- La definición de Química, presentada en la mayoría de textos, no aporta una visión pertinente del objeto de estudio de esta ciencia, al determinar que "La química es una ciencia que estudia la materia y sus transformaciones". De ahí, que el partir del establecimiento de la concepción de cuerpo material, contribuye a la discriminación de las sustancias como objeto de estudio de la química, determinando que un cuerpo material puede ser una sustancia (elemento o compuestol o un conjunto de sustancias (mezclas).

- Todas las sustancias son cuerpos materiales, pero no todos los cuerpos materiales son sustancias, por que estos pueden estar conformados por varias de ellas. Es necesario establecer el concepto de sustancia como un sistema multiatómico, en el que las interacciones entre átomos del mismo tipo (elementos) o de diferente tipo (compuestos). son los que determinan las propiedades características de las sustancias y que permiten diferenciarlas de las mezclas. No se puede emplear la unidad de medida mol, si la materia cuantificada no es una sustancia determinada.

- La razón por la que los estudiantes, al cuantificar la sustancia como cantidad de sustancia, la relacionan con masa o volumen se debe al hecho de considerar que cualquier cuerpo material es una sustancia; y, por lo tanto, confunden cantidad de materia medida por la masa o por el volumen, con la cantidad de sustancia, cuya unidad de medida en el sistema internacional de unidades es el mol. 


\section{0}

\section{Universidad Surcolombiana}

- La validez de las teorías pasadas no puede discutirse con base en los conocimientos actuales, sino a partir del contexto en que surgen. De la misma manera, los esquemas conceptuales de los estudiantes han de analizarse considerándolos como instrumentos que le son útiles para su interpretación e interacción con la realidad. A partir de esto es posible generar la evolución de sus conocimientos.

- Un enfoque sistémico de la construcción de modelos de materia implica un curriculum inicial de Química como sucesión de esquemas conceptuales inclusivos y progresivamente más complejos, delimitados por la naturaleza de los hechos experimentales que son capaces de explicar y predecir.

\section{Contexto teórico en el cual se estructura el concepto Mol}

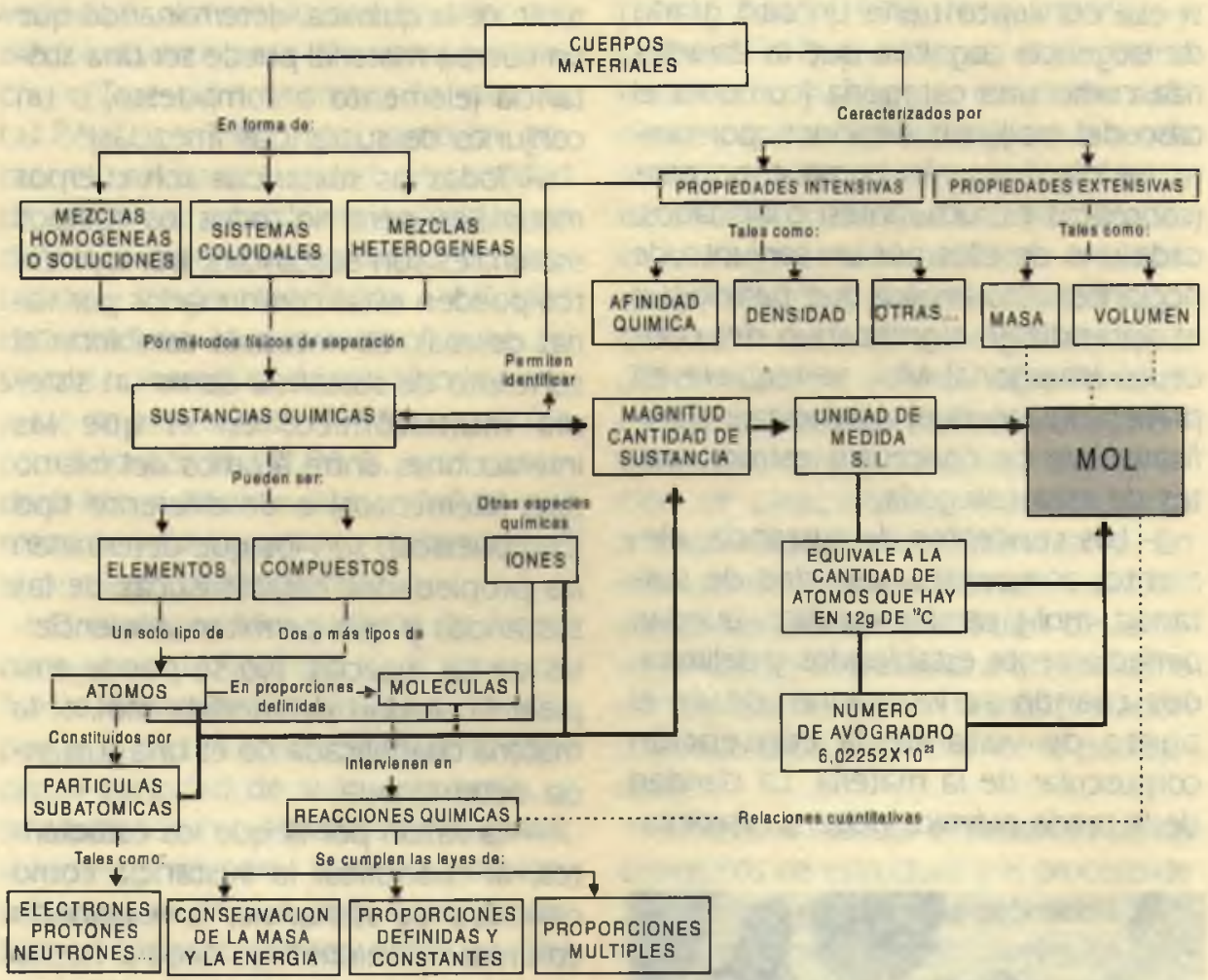


Paideia Surcolombiana

\section{REFERENCIAS BIBLIOGRÁFICAS}

1. ASIMOV. Isaac. 1996. "Breve historia de la Química". Madrid: Alianza Editorial. p. 16-22.

2. COHEN, I. 1991. "Moles and equivalents: Quantities of matter". En: Journal of Chemical Education. V. 68. p. 555-558.

3. CUBILLOS, Germán; POVEDA, Flor; VILLAVECES, José Luis. 1989. "Hacia una historia epistemológica de la Ouímica". Bogotá, D.C: Academia Colombiana de Ciencias Exactas, Físicas y Naturales. p. 53-72.

4. DIEKERS, Werner. 1996. "Teaching the mole". En: European Journal of Science Education. V. 18. p. 145- 154.

5. FURIÓ, C. et al. 1995. "Concepciones de los estudiantes sobre una magnitud no introducida en química: la cantidad de sustancia". En: Enseñanza de las ciencias. V 23. p. 37-39.

6. GARCÍA, J. et al. 1990. "Ideas de los alumnos a cerca del mol". En: Enseñanza de las ciencias, 8 (2). p. 111-119.

7. I.U.P.A.P. 1958. "Recomendation of the International Union of the pure and applied physics. Comission for symbols, units and nomenclature". V. 14. p. 259-268.

8. KOLB, I. 1994. "The mole". En: Journal of Chemical Education. V. 71. p. 728-736.

9. LEE, S. 1961. "A redefinition of mole". En: Journal of Chemical Education. V. 38. p. 549551.

10. LLORENS, Juan. 1991. "Comenzando a aprender química". Mädrid: Visor S.A. p. 47-76.

11. LLORENS. Juan. 1997. "La concepción corpuscular de la materia: obstáculos epistemológicos y problemas de aprendizaje". En: Investigación en la Escuela. No 32. p. 33.

12. NOVICK, S. and MENIS, J. 1996. "Students perceptions of the mole concept". En: Journal of Chemical Education. V. 73. p. 720-722.

13. WURTS, Charles A. 1997. "Teoría de los átomos en la concepción general del mundo". Madrid: Rollo. p. 13-20. 


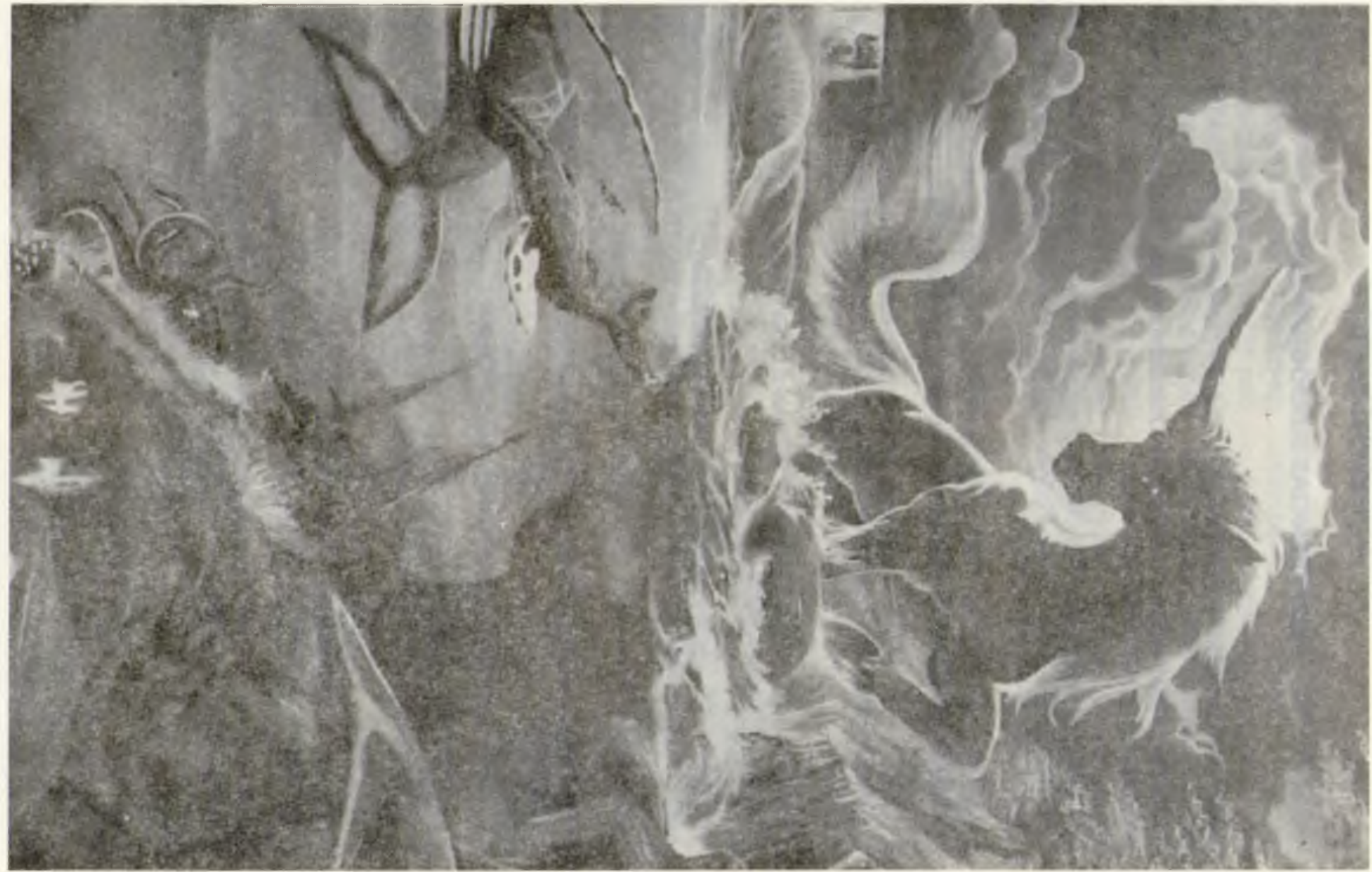

